\title{
Le point sur les cancers cutanés
}

\section{Update on cutaneous cancers}

\author{
C. Gaudy-Marqueste \\ (C) Lavoisier SAS 2018
}

Les cancers cutanés sont de plus en plus fréquents dans le contexte de vieillissement de la population et posent des problèmes de prise en charge spécifiques, liés aux caractéristiques de chaque tumeur. L'oncodermatologie a connu des avancées thérapeutiques majeures au cours des dix dernières années. Pour ce dossier de la revue Oncologie, le Groupe de cancérologie cutanée (GCC) de la Société française de dermatologie (SFD) a choisi de développer les nouveautés physiopathologiques et thérapeutiques de quatre tumeurs : les mélanomes, les carcinomes épidermoïdes, les carcinomes à cellules de Merkel et les carcinomes basocellulaires.

Les articles des Drs Damien Giacchero et Sandrine Monestier $[1,2]$ expliquent comment le développement des inhibiteurs de la voie des MAP kinases et des immunothérapies (anti-CTLA4 et anti-PD1) a conduit à l'approbation de six nouvelles molécules depuis 2011 et a complètement bouleversé la prise en charge des mélanomes métastatiques. La présence d'une mutation somatique du gène $B R A F$ en position V600 a, en effet, ouvert l'accès aux thérapies ciblant la voie des MAP kinases. La stratégie actuelle consiste à associer des inhibiteurs de BRAF et de MEK, puisqu'il a été démontré que cette double inhibition limitait les phénomènes d'échappement observés sous les inhibiteurs de BRAF et en améliorait le profil de tolérance. Deux associations à l'efficacité globalement similaire, mais aux profils de tolérance différents sont actuellement disponibles en première ligne, tandis qu'un troisième couple de BRAF-MEK inhibiteur est en cours d'approbation. Ces traitements ne sont malheureusement accessibles qu'à la moitié des patients, puisque seuls $50 \%$ des mélanomes sont BRAF mutés. Les patients atteints de mélanome métastatique ont également été les premiers à bénéficier des progrès de l'immunothérapie grâce aux antiCTLA4 (ipilimumab) qui permettent d'obtenir des réponses très prolongées chez un petit nombre de patients $(20 \%$ environ). Ces molécules révolutionnaires à l'époque ont été sup-

\footnotetext{
C. Gaudy-Marqueste $(\square)$

Service de dermatologie et de cancérologie cutanée, hôpital Timone, 264, rue Saint-Pierre,

F-13385 Marseille Cedex 5, France

e-mail : Caroline.GAUDY@ap-hm.fr
}

plantées par les anti-PD1, plus efficaces et moins toxiques, qui sont désormais devenus incontournables et peuvent être prescrits aux patients porteurs de mutation ou non. Les associations anti-CTLA4 et anti-PD1, plus efficaces, mais très toxiques, ont une AMM aux États-Unis et en Europe, mais ne sont pas encore accessibles en France. Les effets secondaires immunitaires de ces traitements doivent être bien connus, car potentiellement graves, et leur maniement est délicat, même pour des équipes entraînées. Aujourd'hui, même si les progrès thérapeutiques sont importants et permettent des survies prolongées de certains patients, l'inclusion de nos patients dans les essais thérapeutiques reste une priorité. Les objectifs actuels sont de déterminer les associations ou séquences thérapeutiques les plus efficaces, tout en limitant les toxicités, et surtout d'identifier des biomarqueurs pouvant guider les choix thérapeutiques.

Les progrès thérapeutiques des dernières années ne se sont pas limités au mélanome. L'article du Dr Leblais [3] montre ainsi que l'identification de mutations de PATCH a permis le développement d'inhibiteurs de la voie hedhegog (vismodegib), qui ont aujourd'hui l'AMM pour la prise en charge des carcinomes basocellulaires inopérables et/ou avancés. Les immunothérapies par anti-PD1/PDL1 sont également porteuses d'espoir pour les formes métastatiques de carcinomes épidermoïdes (article du Pr Eve Maubec) [4] et de carcinomes à cellules de Merkel (article du Pr Mahtab Samimi) [5], tumeurs cutanées plus rares, mais au pronostic particulièrement sombre.

Le GCC est un groupe thématique de la SFD qui regroupe l'ensemble des centres français experts en oncodermatologie. Il est actuellement présidé par le Dr Sophie Dalac du CHU de Dijon. Ce groupe fédérateur travaille en lien avec les autorités de santé et l'INCa pour l'élaboration des référentiels concernant la prise en charge diagnostique et thérapeutique des cancers cutanés. Il coordonne plusieurs bases de données sur le mélanome (RicMel et Melbase) et les cancers cutanés rares (CARADERM). Il coordonne également des études cliniques académiques ou en partenariat avec des industriels.

L'ensemble des membres du GCC se joint à moi pour vous souhaiter une bonne lecture, en espérant que cette mise au point répondra à vos attentes. 


\section{Références}

1. Giacchero D (2018) Mélanome : actualités physiopathologiques et stratégie thérapeutique. Oncologie 20: 3-10

2. Monestier S (2018) Mélanome : effets indésirables des traitements innovants. Oncologie 20: 11-17
3. Leblais C (2018) Carcinome basocellulaire : actualités et stratégies thérapeutiques. Oncologie 20: 27-32

4. Maubec E (2018) Carcinome épidermoïde cutané : actualités sur la physiopathologie et les stratégies thérapeutiques. Oncologie 20: $33-36$

5. Samimi M (2018) Carcinome de Merkel : actualités physiopathologiques et thérapeutiques. Oncologie 20: 19-26 